19 Revue d'histoire du XIXe siècle

Société d'histoire de la révolution de 1848 et des

révolutions du XIXe siècle

$36 \mid 2008$

L'enquête judiciaire et ses récits - Mots, violence et politique - Varia

\title{
Eloi Valat, Le Journal de la Commune
}

Préface de Jean-Marie Borzeix, Saint-Pourçain-sur-Sioule/Paris, Bleu autour/Paris bibliothèques, 2007, 151 p. ISBN : 978-2912019677. 24 euros

Jean-Claude Caron

\section{OpenEdition}

Journals

Édition électronique

URL : http://journals.openedition.org/rh19/2912

DOl : $10.4000 /$ rh 19.2912

ISSN : $1777-5329$

Éditeur

La Société de 1848

Édition imprimée

Date de publication : 1 juin 2008

Pagination : 187-189

ISSN : 1265-1354

Référence électronique

Jean-Claude Caron, «Eloi Valat, Le Journal de la Commune », Revue d'histoire du XIXe siècle [En ligne], 36 | 2008, mis en ligne le 05 juillet 2008, consulté le 22 septembre 2020. URL : http:// journals.openedition.org/rh19/2912; DOl : https://doi.org/10.4000/rh19.2912 
Taylor et Michael Wolff en est l'illustration. Si les apports de l'histoire comparée ou croisée entre la Grande-Bretagne et ses voisins non anglophones n'y sont presque jamais évoqués, c'est peut-être que ces travaux utilisent peu la référence à une époque "victorienne». C'est peut-être aussi que cette catégorie issue de la GrandeBretagne impériale du $\mathrm{XIX}^{\mathrm{e}}$ siècle conserve jusqu'à nos jours une connotation anglosaxonne. Impossible à définir autrement qu'en référence au règne de Victoria, elle dessine les contours d'une communauté culturelle sur laquelle le soleil ne se couche jamais, et offre un cadre de réflexion commun entre universitaires de plusieurs disciplines de Santa Barbara à Wellington. Mais on peut se demander dans quelle mesure la catégorisation du XIX ${ }^{\mathrm{e}}$ siècle comme "victorien" n'est pas un frein à une relecture de l'histoire britannique qui articulerait sa dimension anglo-saxonne à sa dimension continentale. Nous autres, Victoriens, sommes aussi des Européens.

\section{Julien Vincent}

Jean-Claude FARCY, Les sources judiciaires de l'époque contemporaine ( $X I X^{e}-X X^{e}$ siècle), "Sources d'histoire», collection dirigée par Nadine Vivier, Paris, Bréal, 2007, 287 p. ISBN : 978-2749507309. 26 euros.

Dans l'introduction de ce livre, Jean-Claude Farcy présente les archives judiciaires d'abord comme objet d'étude historique puis expose leur place comme source dans tous les domaines de l'histoire. Le livre est ensuite découpé en cinq grands thèmes, chacun composé d'une courte introduction problématique : les institutions, le personnel (magistrats et auxiliaires de justice), le fonctionnement de la justice pénale, de la justice civile, enfin les rapports entre justice et société. Chacun de ces thèmes, découpé en quatre dossiers, comporte une courte introduction qui en résume le contenu puis les quatre parties proposées sont explorées à travers deux documents, l'un dont le commentaire est complètement rédigé, l'autre ne comportant qu'un plan de l'exercice. Par exemple, la justice pénale est successivement étudiée à travers un premier dossier intitulé "le rôle du parquet et les filières pénales", appuyé sur un document issu du tribunal de Rambouillet du 2 août 1860 : «un jugement correctionnel à la requête d'une partie civile», suivi d'un deuxième document : un registre des entrées du parquet de Dijon de l'année 1897. Le deuxième dossier du thème porte sur «l'instruction préparatoire», le troisième sur «les assises» et le dernier s'intitule "après le verdict». Cet ouvrage de Jean Claude Farcy nous propose donc un guide précieux et précis des sources judiciaires contemporaines et de leurs usages tant pour les recherches que pour l'enseignement.

\section{Nicole Edelman}

Eloi VALAT, Le Journal de la Commune, préface de Jean-Marie Borzeix, Saint-Pourçain-sur-Sioule/Paris, Bleu autour/Paris bibliothèques, 2007, 151 p. ISBN : 978-2912019677. 24 euros.

Cet ouvrage rend compte de l'exposition des dessins inédits d'Éloi Valat, qui s'est tenue à la Bibliothèque historique de la ville de Paris en mars 2007. La même institution avait déjà, peu de temps auparavant, célébré la Commune de Paris par 
une remarquable exposition consacrée au Regard d'un Parisien sur la Commune ${ }^{39}$, qui avait révélé les étonnants clichés d'Hippolyte Blancard, dont certains constituent des documents exceptionnels sur les incendies de la Semaine sanglante. Le projet d'Éloi Valat est autre. Par le trait et par la couleur, il entend illustrer certains épisodes des 73 jours de la Commune en s'appuyant sur la lecture du Journal officiel de la République française publié par la Commune de Paris entre le 20 mars et le 24 mai $1871{ }^{40}$. Cet organe offre à la fois des informations communiquées par la Commune, essentiellement les délibérations et décisions prises par cette dernière (partie officielle), et des informations variées touchant à toutes sortes de domaines, mais de plus en plus relatives au déroulement de la guerre contre les troupes de Versailles (partie non officielle). L'ouvrage se compose donc de 69 planches illustrant chacune un extrait du Journal officiel, donné en regard. "Des images d'Epinal modernes», commente JeanMarie Borzeix dans sa préface. Certes, mais la dimension des images qui occupent une pleine page est tout autre (à cet égard, il est dommage qu'aucune fiche technique n'accompagne ces illustrations), tout comme differe l'intention de l'artiste : il ne s'agit pas ici d'une dimension pédagogique, moralisatrice, voire ludique, à l'instar des séries éditées par Pellerin à Epinal, mais d'une lecture engagée de l'événement et de ses protagonistes, vus du côté des communards. Il ne s'agit pas non plus d'une bande dessinée à la manière des quatre tomes du Cri du peuple de Tardi et Vautrin, récit mis en image d'une histoire au ressort à la fois politique et policier se déroulant sur fond de Commune. Ce qui est convoqué par Éloi Valat, c'est l'événementCommune, via le prisme de la "grande» comme la "petite» histoire. Ce ne sont pas nécessairement les épisodes les plus célèbres de la Commune qui ont été choisis par l'auteur. Remarquons en particulier le dessin consacré à la tentative de conciliation menée par les francs-maçons parisiens à l'initiative du frère Térifocq (30 avril) ou celui illustrant la séance d'un club féminin (5 mai).

L'événement est perçu et représenté dans toute sa violence, ce dont témoignent à la fois le trait propre à Eloi Valat et le choix d'une palette de couleurs plutôt sombres, sans dégradés, avec des juxtapositions franches. Elles étonnent en confrontant toute la gamme des verts et des bleus à celle des jaunes et des rouges. Le trait de Valat est souvent rétif à la ligne régulière, que ce soit pour le contour des personnages ou pour les lignes de fuite dans les scènes de rue. La netteté de la séparation des masses colorées n'est pas sans rappeler l'art du vitrail. Symboliquement, le dessin illustrant le dernier extrait du dernier numéro du Journal officiel de la Commune ne comporte, outre le trait au noir, que des parements de couleur rouge. Dans les scènes de groupe, les visages sont à peine ébauchés; d'autre signes, nombreux, permettent d'identifier les protagonistes. Influence de l'école allemande faisant la chronique de l'après-Première Guerre mondiale dans un pays en ruine? La composition, le trait, l'intention font à l'occasion penser à Dix, Beckmann ou Grosz (par exemple, le 19 mai). Ici, la profondeur de champ est fournie par un élément urbain (édicule, 22 mars 1871) ; là, le mouvement est donné par la gestuelle des acteurs, y compris un chien (épisode de Thomas et Lecomte) ou une chaise renversée (idem). Les maisons tanguent parfois

39. Jean Baronnet, Regard d'un Parisien sur la Commune. Photographies inédites de la Bibliothèque historique de la ville de Paris, Paris, Gallimard/Paris bibliothèques, 2006.

40. Rappelons la réédition en trois volumes du Journal officiel de la Commune de Paris, Coeuvres-etValsery, Éditions Ressouvenances, 1996. 
comme dans les tableaux de Soutine (25 mars 1871). On remarque la place donnée aux femmes et aux enfants, dont certains évoquent des personnages de Hugo, mais aussi des "poulbots" fin-de-siècle. Certaines scènes donnent à voir plusieurs dizaines de personnages, d'autres sont fondées sur la seule présence d'un bâtiment (Hôtel de Ville) ou d'un drapeau rouge derrière une barricade (31 mars), ou encore sur la perspective en surplomb d'une artère vide (26 mars); d'autres donnent dans la scène de genre, dans le portrait d'inconnus ou, au contraire, de chefs de la Commune (individuellement : Beslay, Courbet, Cluseret, Delescluze, etc., ou en groupe : 7 avril, 6 mai). La présence de la guerre civile est constante, par ses objets - fusils, canons, obusiers et autres armes, passives ou actives - et ses conséquences - ambulances, blessés et mutilés, cadavres de fédérés morts, représentés dans leur cercueil de fortune (10 avril) ou, dans ce qui pourrait former un diptyque, par une série de pieds nus alignés verticalement (23 mai). Image particulièrement étonnante aussi dans sa composition que ce cadavre sans tête (21 avril).

Il convient de saluer la force de cette œuvre. L'histoire, quelle que soit la période considérée, a rarement été "sentie» avec une telle acuité. À sa manière, et même si la démarche est très différente, ce volume évoque le projet cinématographique consacré par Peter Watkins à la Commune ${ }^{41}$. Et illustre qu'histoire et arts ont tout à gagner à se confronter.

Jean-Claude Caron

\section{Jules VALLÈS, Dictionnaire d'argot et des principales locutions popu-}

laires, avant-propos de Maxime Jourdan, Paris, Berg international éditeurs, 2007, 207 p. ISBN : 978-2-917191-00-2. 14 euros.

La langue verte constitue un terrain d'observation particulièrement intéressant quant aux mutations du social, du culturel, voire du politique. Dans son bref, trop bref avant-propos, Maxime Jourdan retrace la généalogie de cet ouvrage qui, rappelle-t-il, est ici publié pour la première fois avec son nom d'auteur : Jules Vallès. Celui-ci avait préféré user d'un de ses habituels pseudonymes, Jean La Rue, lorsqu'il rédigea ce dictionnaire dont la première édition fut publiée en 1894, soit neuf ans après sa mort. Maxime Jourdan donne un argumentaire qui, s'il conclut sur l'attribution de l'œuvre à Vallès, conserve toutefois une part de doute. Pourquoi, du reste, ne pas avoir réédité l'ouvrage sous son titre originel - La Langue verte, dictionnaire d'argot et des principales locutions populaires - et l'avoir amputé du texte liminaire intitulé Histoire de l'argot, par Clément Casciani? Le fait que Vallès ait abhorré la nature même du dictionnaire en général (à la fois comme conservatoire d'une langue figée ou morte qu'il n'a eu de cesse de combattre et de dénoncer et comme représentation par excellence de la rigidité et de la discipline de l'école) ne suffit pas à infirmer la thèse. On sait que Vallès a fait, entre autres métiers alimentaires, celui de "pisse-copie» de notices encyclopédiques pour survivre (voir en particulier la thèse de Maurice Gaudin sur les entreprises lexicographiques de Maurice Lachâ-

41. Peter Watkins, La Commune (Paris, 1871). Première version : 1999, 5h 45; deuxième version : $2007,3 \mathrm{~h} 30$. 